Int. J. Morphol.,

31(3):967-972, 2013.

\title{
Inervación de las Regiones Escapular, Braquial y Antebraquial de la Llama (Lama glama)
}

\author{
Innervation of the Scapular, Brachial and Antebrachial of the Llama (Lama glama)
}

Carlos A. Arzone*,**; Pablo Genoud*,**; Fernando C. Pellegrino* \& Ramiro J. Vidal Figueredo*

ARZONE, C.A.; GENOUD, P.; PELLEGRINO, F. C. \& VIDAL, F. R. J. Inervación de las regiones escapular, braquial y antebraquial de la llama (Lama glama). Int. J. Morphol., 31(3):967-972, 2013.

RESUMEN: La llama (Lama glama) es un mamífero cuya producción ha crecido exponencialmente en las últimas décadas, sobre todo en la región noroeste y patagónica de Argentina, Chile, Bolivia y Perú. Asociada a este crecimiento, la clínica médica veterinaria ha tenido que enfrentarse con el desafío de nuevos diagnósticos neuroanatómicos, así como también con maniobras semiológicas y quirúrgicas específicas para esta especie. El objetivo del presente trabajo es realizar una detallada descripción de la inervación de las regiones de la espalda, el brazo y el antebrazo, aportando conocimientos que sean de utilidad para que el veterinario clínico pueda interpretar alteraciones funcionales o para la realización de tratamientos quirúrgicos, terapéuticos y/o diagnósticos. Se incluye además la descripción de las relaciones musculares y vasculares de los nervios.

PALABRAS CLAVE: Lama; Inervación del miembro torácico; Relaciones musculares; Relaciones vasculares.

\section{INTRODUCCIÓN}

El objetivo del trabajo es realizar una detallada descripción del plexo braquial y de los nervios que de él se originan, especialmente aquellos relacionados a la cintura y miembro torácicos. Debido a que la región de la mano, por su complejidad, merece un estudio particularizado, no ha sido objeto de estudio en el presente trabajo.

La llama pertenece a la infraclase de los euterios ferungulata-, cohorte ungulados -Ungulata- orden artiodáctilos -Artiodactila-, es en este último punto de la clasificación que es importante detenerse pues al investigar la distribución nerviosa periférica de una especie desconocida se impuso la necesidad de compararlo con una especie doméstica de la cual se tiene acabado conocimiento como lo es el bovino (Cabrera, 1932; de Lamo, 2011; Wheeler, 1995). Como objetivo secundario se propuso la comparación neuroanatómica del miembro torácico de la llama con la del bovino. La razón de lo expuesto se fundamenta en que los rumiantes, también pertenecen al orden de los artiodáctilos y por ende con los que se buscó establecer diferencias y similitudes en una forma lógica. Los datos obtenidos de las descripciones realizadas sobre camellos por Lesbre, nos permitieron realizar una comparación con una especie más cercana taxonómicamente a la llama (Lesbre, 1903a, 1903b).
El importante impulso que alcanzó la producción de camélidos sudamericanos en la última década, con el propósito de obtener carne y pelo, tanto en forma natural, como en explotaciones intensivas, ha convertido a esta especie en una nueva fuente de trabajo para el profesional veterinario. El aporte al conocimiento de la inervación del miembro torácico va más allá de la simple descripción anatómica, tiene como meta contribuir al saber del veterinario para que este sea capaz de realizar diagnósticos neurológicos, neurectomías, neuroestimulación o evitar su lesión en cirugías de las regiones que nos ocupan. Uno de los pilares básicos de la práctica veterinaria indica que toda aproximación clínica debe indefectiblemente partir del conocimiento anatómico para ubicar las estructuras, esta premisa realza su importancia en la neuroanatomía. Se ha incluido el territorio de inervación y las relaciones de los principales troncos nerviosos. Otros autores han abordado el tema en especies evolutivamente cercanas a la llama; en el libro "Medicine and Surgery of South American Camelids" se cita en el capítulo de sistema nervioso un ejemplo de paraparesia, como consecuencia de una lesión del nervio radial debido a una prolongada exposición lateral durante una anestesia. El mismo autor reconoce que colocando un almohadón en lateral del miembro durante el decúbito, dicho suceso puede evitarse (Fowler, 1998).

\footnotetext{
* Cátedra de Anatomía, Facultad de Ciencias Veterinarias, Universidad de Buenos Aires, Argentina.

** Cátedra de Anatomía, Carrera de Medicina Veterinaria, Universidad Nacional de Río Negro, Argentina.
} 
Sato et al. (1983), en la Universidad Mayor de San Marcos (1975-1979), describe la anatomía de los nervios del plexo axilar de la alpaca (Lama pacos) junto con la inervación de la región del hombro y el brazo.

\section{MATERIAL Y MÉTODO}

Las disecciones se realizaron sobre especímenes de llama (Lama glama) provenientes de la provincia de La Pampa. Se utilizaron tres llamas adultas de descarte, dos machos y una hembra. Los animales fueron premedicados con Xilacina (Romúm ${ }^{\circledR}$ Bayer) en dosis de $1 \mathrm{mg} / \mathrm{Kg}$ por vía intramuscular. Posteriormente se procedió a anestesiarlos con pentotal sódico (Abott) vía endovenosa. Una vez en plano anestésico se procedió al sangrado "a blanco". Finalizado el proceso se disecó la arteria carótida común para canalizarla y luego perfundir una solución fijadora de formol al 7\% y acido fénico al $2 \%$ en solución acuosa.

Los animales fueron acondicionados y remitidos a cátedra de Anatomía de la Universidad de Buenos Aires, donde fueron cuidadosamente cuereados y colocados en piletas con una solución de formol al $3 \%$ y acido fénico al $1 \%$ para su conservación.

La disección se realizó con instrumentos convencionales, el material fue periódicamente rociado con una solución de acido fénico, alcohol y glicerina para evitar su desecación. Una vez aislados los nervios se colorearon con pintura acrílica amarilla (Acrimax®).

\section{RESULTADOS}

En todos los especímenes hallamos la presencia de ocho nervios que se originan desde el plexo braquial -plexus brachialis- y se incorporan a las regiones de la cintura escapular y miembro torácico de la llama. Los mismos son:

Nervio supraescapular-N. suprascapularis-

Nervios subescapulares-N. subecapulares-

Nervio toracodorsal-N. thoracodorsalis-

Nervio axilar-N. axilaris-

Nervio radial-N. radialis-

Nervio musculocutáneo-N.musculocutaneus-

Nervio ulnar-N. ulnaris-

Nervio mediano-N. medianus-

A continuación se presentan los hallazgos y descripciones de cada uno:
Nervio supraescapular - $N$. suprascapularis. Es un grueso nervio, que proviene de la porción craneal del plexo braquial, se dirige desde su origen en dirección lateroventral, cruzando la cara medial del músculo supraespinoso $-m$. supraspinatus-, se relaciona en medial con la arteria supraescapular -a. suprascapularis-, para ubicarse en el espacio entre el músculo supraespinoso y el músculo subescapular $-m$. subscapularis-. A unos $5 \mathrm{~cm}$ por dorsal de la articulación humeral -articulatio humeri-, rodea el margen craneal de la escápula, en profundidad del músculo supraespinoso, pasa hacia la cara lateral girando alrededor del cuello de la escápula, siempre acompañado por la arteria y la vena supraescapular conformando así un paquete vasculonervioso supraescapular. En ese sitio se encuentra una almohadilla adiposa, que protege al nervio y que incluye también a los vasos sanguíneos. Sobre la cara lateral de la región se dirige caudalmente, rodeado por la espina de la escápula por ventral y cubierto por el acromion. A esa altura toma una dirección ascendente ubicándose entre el acromion y el músculo infraespinoso - m. infraspinatus- sobre el cual termina ramificándose. Su función es inervar el músculo supraespinoso e infraespinoso. (Fig. 1).

Nervio subescapular - N. subscapularis-. Se origina caudalmente al nervio precedente. Tras un muy corto trayecto se ramifica presentando entre dos y cuatro ramos muy delgados, que cruzan caudalmente la superficie del músculo subescapular, al cual inervan. Cabe destacar que el músculo subescapular recibe una delgada rama proveniente del nervio axilar, esta última no debe confundirse con otro ramo del nervio subescapular (Fig. 1).

Nervio toracodorsal $-N$. thoracodorsalis-. Este nervio se ubica por medial del nervio axilar, se dirige oblicuamente hacia caudal cruzando la superficie medial de la espalda en el espacio entre el músculo m. supraespinoso, la inserción del m. subescapular y los vasos subescapulares, el tercio medio del músculo redondo mayor $-m$. teres major-, para alcanzar la cara profunda del $\mathrm{m}$. latísimo del dorso $-m$. latissimus dorsi-, sobre el cual se ramifica. Lo acompañan en su trayecto los vasos toracodorsales, formando el paquete neurovascular toracodorsal sobre la superficie medial del músculo latísimo del dorso (Fig. 1).

Nervio axilar $-N$. axilaris-. Su origen en el plexo es caudal al nervio musculocutáneo, cruza la porción distal del musculo subescapular, alcanzando la superficie caudal de la articulación humeral. En su recorrido destina un ramo importante para el músculo subescapular y otra que penetra en el músculo redondo mayor $-m$. teres major-. Una vez ubicado en el hueco formado por los músculos redondo mayor, subescapular, coracobraquial-m. coracobrachialis, la articulación humeral y la cabeza larga del músculo tríceps 
braquial-caput longum, m. tríceps brachii-, pasa a través de él, de medial a lateral. El nervio axilar se localiza luego sobre la cara lateral de la región braquial, para su mejor visualización se secciona la porción acromial del músculo deltoides -pars acromialis, m. deltoideus. En este lugar emite un ramo que pasando entre éste y la cabeza lateral del músculo tríceps braquial, se dirige cranealmente para finalizar inervando al músculo cleidobraquial-m. cleidobrachialis-. Aquí se observa además, un delgado ramo cutáneo, el nervio cutáneo craneal del antebrazo - n. cutaneus antebrachii cranialis-, que se distribuye por la fascia de la cara craneal del antebrazo, alcanzando la piel de la región. No se ha observado la presencia del nervio cutáneo craneal lateral del brazo $-n$. cutaneus brachii lateralis cranialis-. Es importante destacar que en el hueco axilar, el nervio se encuentra acompañado por la arteria circunfleja humeral caudal $-a$. cincunflexa humeri cranialis- y la arteria colateral radial a. collateralis radialis- (Fig. 1).

Nervio radial- $N$. radialis-. Como sucede en otras especies domésticas, es el ramo más gruesa del plexo braquial. Cruza la cara medial de la arteria braquial - a. brachialis- para dirigirse en dirección caudal y relacionarse con el nervio ulnar $-n$. ulnaris-. Pasa luego hacia lateral de la región del brazo, en el intersticio entre el músculo redondo mayor y las cabezas larga y medial del músculo tríceps braquial. Durante un corto trayecto se encuentra acompañado por ramas de la arteria braquial profunda -a. brachialis profunda. Acompañando al músculo braquial, se dirige hacia la cara lateral del antebrazo cubierto por la cabeza lateral del músculo tríceps braquial, la cual debe ser seccionada para tener una mejor visualización del nervio. En este trayecto destina ramos que se introducen en las cabezas del músculo tríceps braquial y en el músculo ancóneo-m. aconeus-. Cerca del margen distal de la cabeza lateral del tríceps braquial, se desprende un ramo, el ramo superficial -ramus superficialis, que tal como sucede en porcinos y caninos, derivan de él, un ramo medial -ramus medialis- y otro lateral -ramus lateralis-, los cuales se sitúan paralelamente a uno y otro lado de la vena cefálica -v. cephalica-.

El tronco principal del nervio continúa como un ramo profundo -ramus profundus-, que alcanza a todos los músculos extensores de carpo y dedo, incluyendo al extensor ulnar del carpo, el cual también se halla desplazado hacia el grupo flexor, como en bovinos y equinos (Fig. 1).

Nervio musculocutáneo-N. musculocutaneus-. Desciende desde su origen pasando por la cara lateral de la arteria axilar, en distal de la misma una pequeña cantidad de fibras se

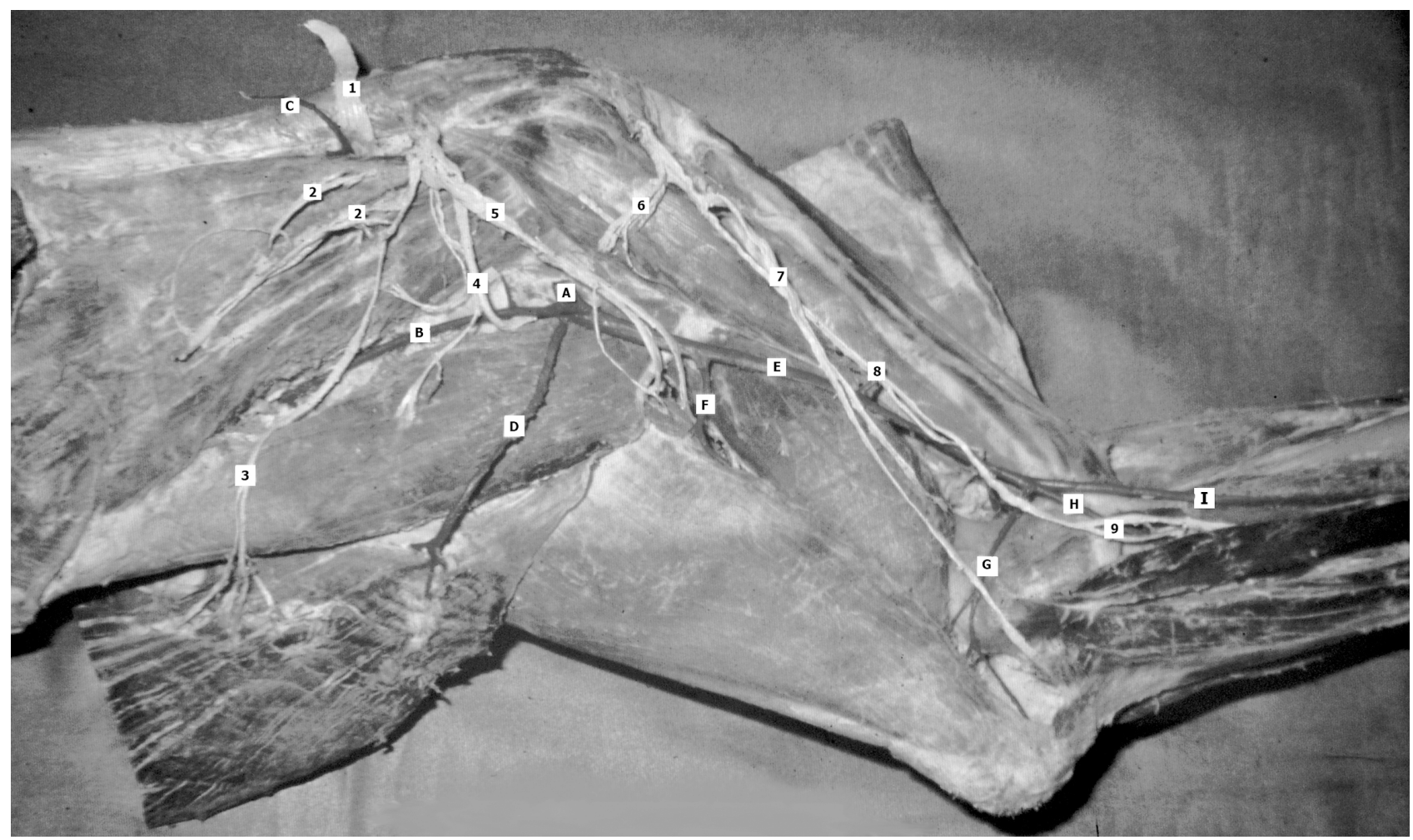

Fig. 1. Vista medial de la región escapular, braquial y antebraquial, mostrando las estructuras nerviosas (N) y arteriales (A). 1- N. axilar; 2- N. subescapular; 3- N. toracodorsal; 4- N. supraespinoso; 5- N. radial; 6- N. musculocutáneo; 7- N. ulnar; 8- N. mediano; A- A. axilar; B- A. subescapular; C- A. supraescapular; D- A. torácica lateral; E- A. braquial; F- A. colateral radial; G- A. ulnar; H- A. interósea común; I- A. mediana. 
intercambian con el nervio mediano, para constituir un frágil lazo alrededor de la arteria, conformando lo que en ungulados se conoce como el ansa axilar -ansa axilaris-. En la región medial del antebrazo se haya envuelto junto al nervio mediano en una vaina de tejido conectivo que dificulta su individualización. Un grupo de fibras se mantiene independiente e inmediatamente en distal de la articulación humeral dichas fibras forman el ramo muscular proximal ramus muscularis proximalis-. Éste pasa entre las dos porciones del músculo coracobraquial-m. coracobrachialis-al cual inerva. Luego se dirige lateralmente para continuar su recorrido cranealmente, penetrando en el sitio en que se dividen los dos vientres del músculo bíceps braquial $-m$. bíceps brachialis-, donde finaliza. La llama, a diferencia de otras especies domésticas, no presenta una arteria circunfleja humeral craneal $-a$. cincunflexa humeri cranialis- satélite que acompañe a esta rama del nervio. En el tercio proximal de la región del brazo se relaciona caudalmente con la arteria braquial y medialmente con la vena homónima. En el tercio medio del brazo desprende un ramo, el ramo muscular distal-ramus muscularis distalis- que se dirige directamente a inervar el músculo braquial (Fig. 1).

Nervio ulnar- N. ulnaris-. Se origina de la parte más caudal del plexo braquial, desciende cruzando oblicuamente la región del brazo en dirección a la superficie medial de la articulación cubital, relacionándose lateralmente con los vasos braquiales. En el tercio medial del brazo se desprende un ramo cutáneo, el nervio cutáneo caudal del antebrazo - $n$. cutaneus antebrachii caudalis- que se distribuye en la fascia que cubre la superficie caudomedial de la cabeza mayor del tríceps, llegando a la piel de la parte caudal del antebrazo. El tronco principal, cruza el epicóndilo medial del húmero donde toma contacto con los vasos colaterales ulnares para ubicarse entre las cabezas de origen del músculo extensor ulnar del carpo-m. extensor carpoulnaris- y del músculo flexor carpoulnar-m. flexor carpo ulnaris-. Desciende entre ellos a lo largo de toda la región caudal del antebrazo; en proximal de la misma destina ramos musculares para los músculos extensor y flexor carpo ulnar, flexor digital superficial, flexor digital profundo y carporradial.

En proximal al hueso accesorio -os carpi accessorium-del carpo en nervio ulnar emite una rama superficial que pasa entre los tendones de inserción de los músculos extensor ulnar del carpo y flexor digital superficial, para ramificarse en la superficie lateral de la región carpiana. La continuación del nervio hacia distal está representada por un delgado ramo que se ramifica sobre la superficie lateral del canal del carpo (Figs. 1 y 2).

Nervio mediano $-\boldsymbol{N}$. medianus-. Desciende sobre la cara medial de la arteria braquial, compartiendo una misma vaina de tejido conectivo con el nervio musculocutáneo. Este nervio se reconoce fácilmente pues en todo su recorrido acompaña a los troncos arteriales más importantes del miem-



Fig. 2. Vista de la cara medial de la región antebraquial del miembro torácico de la llama, señalando los nervios $(\mathrm{N})$, las arterias $(\mathrm{A})$ y músculos (M). 7- N. ulnar; 8- N. mediano; I- A. mediana; m. ECR: M. extensor radial del carpo; m. FCR: M. flexor radial del carpo; m.FPD: M. flexor digital profundo; m. Trc: M. tríceps braquial (cabeza mayor y cabeza medial); m. L F: lacerto fibroso del M. bíceps braquial. 
bro torácico. Alcanza la articulación cubital, donde se sitúa entre la inserción del músculo bíceps braquial y el origen de la poderosa cabeza del músculo flexor digital profundo- $m$. flexor digitorum profundus-. Aquí se ubica superficialmente, junto con la arteria mediana y con la red venosa que conforman, hacia proximal las venas medianas. En el tercio proximal del antebrazo, emite los ramos musculares para los vientres de los músculos flexores de carpo y de los dedos, estos ramos acompañan en general a la arteria interósea común - $a$. interossea communis-. El nervio mediano continúa distalmente primero entre la cabeza radial del músculo flexor digital profundo y el músculo flexor carpo radial, para ubicarse luego entre este último y el radio, distalmente continúa acompañado por los vasos medianos, y con ellos ingresa a la región del carpo (Figs. 1 y 2).

\section{DISCUSIÓN}

Es escasa la bibliografía descriptiva referida a la neuroanatomía de los camélidos y aún más la que enfoca su estudio en los camélidos sudamericanos. Es llamativa la similitud hallada entre los textos descriptivos del Dr. F. Lesbre, respecto a los camélidos del viejo mundo y los ha- llazgos en nuestras disecciones. Citaremos por ejemplo la presencia del ansa axilar en la llama, conformada por el entrecruzamiento de fibras entre el nervio musculocutáneo y el nervio mediano, en los escritos del Dr. Lesbre hallamos la descripción "el nervio braquial [n. musculocutáneo] se anastomosa con el nervio mediano por ventral de la arteria humeral [arteria braquial]". Aunque no hay una detallada descripción, el mismo autor relata la presencia de los nervios supraescapular, subescapular, axilar, radial y ulnar.

Por otro lado hemos comparado las descripciones realizadas por otro autores (Getty, 1975; Nikel et al., 1984; Schwarze \& Schroeder, 1970; Sisson \& Grossman, 1961) y especialmente hemos tenido en cuenta la Nomina Anatomica Veterinaria (N. A. V.) que cita cada una de los ramos de los nervios del plexo braquial en las diferentes especies, en este caso hemos tenido como esquema principal la descripción para rumiantes. Sus nervios y ramos se detallan en la Tabla I.

Se puede observar que no existen diferencias significativas entre las descripciones de la N. A. V. respecto a ungulados y nuestros hallazgos en las disecciones de la inervación de llamas en las regiones de la espalda, brazo, antebrazo y carpo.

Tabla I. Ramos de los nervios del plexo braquial en las ungulados y llama (Lama glama).

\begin{tabular}{|c|c|}
\hline N.A.V. Ungulados & Hallazgos en la llama \\
\hline N. supraescapular & N. supraescapular \\
\hline Nn. subescapulares & Nn. subescapulares \\
\hline N. musculocutáneo & N. musculocutáneo \\
\hline - Ramo muscular proximal & - Ramo muscular proximal \\
\hline - Ansa axilar & - Ansa axilar \\
\hline - $\quad$ Ramo muscular distal & - $\quad$ Ramo muscular distal \\
\hline N. Axilar & N. Axilar \\
\hline - $\quad$ Ramos musculares & - $\quad$ Ramos musculares \\
\hline - $\quad$ N. cutáneo lateral craneal del brazo & - $\quad$ N. cutáneo lateral craneal del brazo \\
\hline - $\quad$ N. cutáneo antebraquial medial & - $\quad$ N. cutáneo antebraquial medial \\
\hline N. Radial & N. Radial \\
\hline - $\quad$ Ramos musculares & - $\quad$ Ramos musculares \\
\hline - $\quad$ Ramo profundo & - $\quad$ Ramo profundo \\
\hline$\circ \mathrm{R}$ amos musculares & $\circ$ Ramos musculares \\
\hline - $\quad$ Ramo superficial & - $\quad$ Ramo superficial \\
\hline ○ N . cutáneo lateral del antebrazo & N. cutáneo lateral del antebrazo \\
\hline ○ $\mathrm{R}$ amo lateral (Car, Su) & Ramo lateral \\
\hline o $\mathrm{R}$ amo medial (Car, Su) & $\circ \quad$ Ramo medial \\
\hline N. Mediano & N. Mediano \\
\hline ○ $\mathrm{R}$ amos Musculares & Ramos musculares \\
\hline ○ $\mathrm{N}$. interóseo del antebrazo & \\
\hline N. Ulnar & N. Ulnar \\
\hline ○ N . cutáneo caudal del antebrazo & N. cutáneo caudal del antebrazo \\
\hline ○ $\mathrm{R}$ amos musculares & Ramos musculares \\
\hline
\end{tabular}




\section{CONCLUSIÓN}

Como en todos los vertebrados, el plexo braquial de la llama se forma por la confluencia de las ramas ventrales de los últimos nervios espinales cervicales y los primeros torácicos. El acabado conocimiento de la anatomía y la distribución de sus componentes nerviosos son de fundamental importancia para la práctica clínica y quirúrgica en esta especie. Conocerlos, permitirá experimentar la realización de bloqueos anestésicos e intervenciones quirúrgicas; tam- bién posibilitará la realización de maniobras indoloras en las diferentes regiones del miembro torácico, como por ejemplo el examen de los dedos y los espacios interdigitales. El profundo conocimiento de los músculos inervados por las diferentes ramas nerviosas facilitará la interpretación de signos clínicos de origen neuromuscular presentes en patologías del miembro torácico e identificar lesiones neurológicas. Para los semiólogos constituye una herramienta fundamental ya que conocer la neuroanatomía es el recurso básico para poner en práctica las pruebas semiológicas o evaluar reflejos miotáticos en el miembro torácico.

ARZONE, C. A.; GENOUD, P.; PELLEGRINO, F. C. \& VIDAL, F. R. J. Innervation of the scapular, brachial and antebrachial of the llama (Lama glama). Int. J. Morphol., 31(3):967-972, 2013.

SUMMARY: The llama (Lama Glama) is a mammal whose production has grown exponentially in the last decades, especially in the Northwest and Patagonian region of Argentina, Chile, Bolivia and Peru. Associated with this growth, veterinary medical clinic has had to deal with the challenge of new neuroanatomical diagnostic, as well as surgical and semiological maneuvers specific to this species. This work aims to make a detailed description of the innervation of the regions back, arm, and forearm, providing knowledge that is useful to the clinical veterinarian to interpret functional alterations or for the performance of surgical, therapeutic, treatment and/ or diagnosis. It also includes the description of the muscular and vascular relationships of nerves.

KEY WORDS: Llama; Innervation of forelimb; Muscular relation; Vascular relation.

\section{REFERENCIAS BIBLIOGRÁFICAS}

Cabrera, A. Sobre los camélidos fósiles y actuales de la América austral. Rev. Mus. La Plata, 33:89-117, 1932.

de Lamo, D. A. Camélidos sudamericanos: Historia, usos y sanidad animal. Buenos Aires, SENASA, 2011.

Fowler, M. E. Medicine and Surgery of South American Camelids: Llama, Alpaca, Vicuna, Guanaco. $2^{\text {nd }}$ ed. Iowa, Wiley-Blackwell, 1998.

Getty, R. Sisson and Grossman's. The anatomy of the domestics animals. $5^{\text {th }}$ ed. Philadelphia, Saunders, 1975.

Lesbre, F. X. Notes sur quelques dispositions anatomiques. Inédites peu connues contatées chez les Camélidés et le Porc-epiccommun. C. R. Ascanat, 1903a. pp.196-7.

Lesbre, F. X. Recherches anatomiques sur les camélidés. Anatomie du chameaux a deux bosses; differences entre les deux espaces de chameaux; differences entre les chameaux et lexlamax. Arch. Mus. Ist. Nat. Lyon, 8-196, 1903b.

Nickel, R.; Schummer, E.; Seiferle, E. The anatomy of the domestic animal. Vol IV. The nervous system, the endocrine glands and the sensory organ of the domestic mammals. Berlin, Verlag Paul Parey, 1984.

Sato A, Kitsutani G, Guzmán J. Inervacion periferica de los segmentos hombro y brazo de la alpaca (Lama pacos ). Lima, UNMSM, 2:44, 1983.
Schwarze, E. \& Schroeder, L. Compendio de Anatomía Veterinaria. Tomo III. Zaragoza, Acribia, 1970.

Sisson, S. \& Grossman, D. D. Anatomía de los Animales Domésticos. Barcelona, Salvat, 1961.

Wheeler, J. C. Evolution and present situation of the South American Camelidae. Biol. J. Linn. Soc., 54(3):271-95, 1995.

Dirección para Correspondencia:

Dr. Vet. Ramiro José Vidal Figueredo

Área de anatomía

Facultad de Ciencias Veterinarias

Universidad de Buenos Aires

Argentina

Email: rvidalfigueredo@hotmail.com

Recibido: 07-08-2012

Aceptado: 29-04-2013 